\title{
ESTABELECIMENTO DE CONDIÇÕES OPERACIONAIS \\ PARA O BICO ROTATIVO MICROMAX NA APLICAÇÃO DO HERBICIDA GLYPHOSATE*
}

\author{
P.C.R. OLIVEIRA* \\ T. MATUO** \\ * Estagiário da FCAV-UNESP \\ ** Professor-Assistente-Doutor da FCAV- \\ UNESP 14870 - Jaboticabal, SP. \\ Parte do Trabalho de Graduação \\ apresentado pelo primeiro autor a \\ Faculdade de Ciências Agrárias e \\ Veterinárias - UNESP.
}

\section{RESUMO}

Alguns parâmetros operaciona is do bico rotativo Micromax para apli cação do herbicida glyphosate foram estudados em condições de labor atório. O bico Micromax a $1.600 \mathrm{rpm}$ e vazão de 0,96 $1 / \mathrm{min}$ forneceu gotas com vmd de $280 \mu \mathrm{m}$ e à vazão de $0,43 \mathrm{i} / \mathrm{min}$, de $232 \mu \mathrm{m}$, sendo que em ambas as condições o coeficiente de dispersão $(\mathrm{r}=\mathrm{vmd} / \mathrm{nmd})$ foi inferior a 1,4 , atendendo, portanto, às especificações para o processo CDA. A distância entre bicos a serem montadas numa barra foi determinada em mesa de estudo do padrão de deposi- ção foi de $1,80 \mathrm{~m}$ para a vazão de 0,96 $\mathrm{Z} / \mathrm{min}$ e concentração da formulação comercial do glyphosate entre $4 \%$ e $6 \%$. Essa distancia foi de $1,40 \mathrm{~m}$ para vazão de $0,43 \quad 1 / \mathrm{min}$ e concentração de glyphosate entre $9 \%$ e $13 \%$. Distancias fora dessas especificações produziram deposições bastante irregulares sob a barra de pulverização.

Palavras-chave: CDA, bico centrifugo, bico rotativo padrão de depos ição, pulv erização. 


\section{SUMMARY}

$\begin{array}{lrr}\text { ESTABLISHMENT } & \text { CF } & \text { OPERATIONAL } \\ \text { CONDITIONS FOR } & \text { MICROMAX } \\ \text { ROTARY ATOMIZERS } & \text { IN } & \text { THE } \\ \text { APPLICATION OF GLYPHOSATE. } & \end{array}$

Some operational parameters of Micromax rotary atomizers in the application of glyphosate were studied under laboratory conditions. Micromax nozzle at $1.600 \mathrm{rpm}$ and $0,961 / \mathrm{min}$ feed rate produced $280 \mathrm{~m}$ vmd droplets and $0,431 / \mathrm{min}$ feed rate, produced $232 \mathrm{~m}$ vmd droplets. In both conditions the dispersion coefficient $(\mathrm{r}=$ $\mathrm{vmd} / \mathrm{nmd}$ ) was bellow 1,4 , attending to the sped fication for CDA. Nozzle spacement between Micromax heads in a spray boom was established as $1,80 \mathrm{~m}$ for $0,961 / \mathrm{min}$ feed rate using $4 \%$ to $6 \%$ glyphosate (commercial) solution and $1,40 \mathrm{~m}$ for $0,43 \mathrm{Z} / \mathrm{min}$ feed rate using $9 \%$ to $13 \%$ solution. Nozzle spacement out of these specifications produces very irregular deposition patterns.

Key words: CDA, centrifugal nozzle, rotary atomizer, deposition pattern, spray

\section{INTRODUÇÃO}

Em 1975, J.D. Fryer, diretor da Weed Research Organization ( WRO ) propôs a sigla CDA, iniciais da expressão "Controlled drop application", para caracterizar uma aplicação na qual as gotas utilizadas e- ram bastante uniformes (11). Em 1978 foi realizado o Simpósio sobre CDA, na Universidade de Reading, In glaterra, no qual os princípios des se processo foram estabelecidos. Atualmente CDA indica a "produção e aplicação de gotas de tamanho ade quado ao controle, com pequena variação no tamanho delas, independente do equipamento e para qualquer volume de aplicação" $(4,5)$. Johnstone (8) propôs que o coeficiente de dispersão (r) das gotas, representado pela razão entre o diâmetro mediano volumétrico (vm- volume median diameter) e o diâmetro mediano numérico (nmd- number median diameter), deve ser menor que 1,4 para caracterizar uma aplicação pelo processo CDA. Mais recentemente (1) foi proposto que junto com a sigla CDA, seja utilizado um índice para dar ideias do tamanho das gotas utilizadas. Assim, CDA 250 indica o emprego de gotas de 250 um de vmd. O método CDA pode ser praticado por qualquer equipamento que produza as gotas cuja uniformidade se enquadre dentro do critério estabelecido $(r<1,4)$ e que estejam apropriadas as finalidades do controle pretendido. Entretanto, na prática, a sigla CDA esta exclusivamente associada, erroneamente, a aplicação por meio de bicos centrífugos.

Bals (2) determinou a variação do tamanho de gotas produzidas pelos bicos rotativo e hidráulico e constatou que o bico rotativo Battleship (antiga denominação do bico Micromax) operado a $2000 \mathrm{rpm}$ e 
vazão de $1,0 / / \mathrm{min}$ produz gotas de $250 \mathrm{~m}$ de vmd e $170 \mathrm{~m}$ de nmd, com r igual a 1,47, enquanto que o bico hidráulico 8002 LP operado à pres- são de 1 bar e vazão de 0,7 $1, /$ min, produz gotas de vmd de $273 \mathrm{~m} \mathrm{e}$ nmd de $38 \mathrm{~m}$, com $\mathrm{r}$ igual a 7,18. No bico rotativo, apenas $6,6 \%$ das gotas eram menores que 50 micra ao passo que no bico hidráulico, a proporção de gotas menores que 50 micra era de 58,7\%. As gotas menores que 50 micra são justamente as mais sujeitas à deriva.

O padrão de distribuição do bico rotativo do ponto de alimentação dos discos, sendo assimétrico se alimentado por um só ponto $(9,6,7)$. É pois, importante que a alimentação do disco seja feita uniformemente através de vários pontos.

A eficiência do bico, quando es tes estão montadas em uma barra pulverizadora, também é função da distância entre eles. Heijne (6) estudou o padrão de deposição de diferentes bicos e conclui que, como existem diferentes padrões de distribuição para diferentes condições operacionais do bico, a distância entre os menores deve ser previamente estudada antes da sua montagem na barra de aplicação.

O presente trabalho teve como objetivo estudar as condições de operação dos bicos rotativos Micro-max na aplicação do herbicida glyphosate para que o padrão de distribuição seja o mais uniforme possível sob a barra de pulverização.

\section{MATERIAL E MÉTODOS}

A distancia entre bicos foi deter minad a com auxílio de uma mesa de canaletas para a determinação do padrão de deposição, com 4,0m de comprimento e 2,5 $\mathrm{m}$ de largura, com as canaletas espaçadas de $6,7 \mathrm{~cm}$ en tre si, no sentido da largura e com uma inclinação de 13 graus. Dois bicos Micromax* foram fixados em suporte sobre a mesa onde a distância entre eles podia ser regul ada. O líquido em teste era coloc ado no reservatrorio de 8,5 litros onde era injetado ar comprimido proveniente de um compressor de ar, de forma a produzir vazão de $0,96 \mathrm{l} / \mathrm{min}$ para o restritor $\mathrm{n}^{0} 55$ e de $0,431 / \mathrm{min}$ para o restritor $\mathrm{n}^{\circ} 37$. 0 líquido pulverizado sobre a mesa de canaletas era coletado em tubos graduados e a leitura trans posta para o papel milimetrado para a conf ecção dos gráficos. A rotação do bico Micro max, regulado para a posição de baixa rotação, foi medida através do tacômetro de vibrações (Vibratak) que forneceu a leitura de $1.600 \mathrm{rpm}$, traba lhand o sem a carga de líquido.

Os tamanhos das gotas produzidas, com o empre go de solução de glyphosate** nas concentrações de $4 \%, 5 \%$ e $6 \%$ do produto comercial, para o restritor $\mathrm{n}^{\circ} 55$ e $9 \%, 11 \%$ e $13 \%$ para o restritor $\mathrm{n}^{\circ} 37$, com o disco a $1600 \mathrm{rpm}$ e pressão suficiente para produzir as vazões citadas, foram determinados. As gotas 
foram coletadas em placas de Petri de $5 \mathrm{~cm}$ de diâmetro contendo matiz graxa (12) e medidas em microscópio óptico Olympus acoplado ao monitor de vídeo National.

\section{RESULTADOS E DISCUSSÃO}

O padrão de distribuição individual do bico Micromax pode ser observado na Figura 1 A. Esse bico e dotado de dois tubos alimentares, e portanto sua distribuição ocorreu simetricamente ao ponto central, igualmente ao citad o por Lake et al. (9), Heijn e (6) e Johns tone (8), o que não ocorre com bicos dotados de apenas um tubo alimentador (6). Algumas curvas representativas do padrão de distribuição, quando feita a sobre posição de dois bicos, po dem ser observado nas Figuras de 1 a 3. Para cada distância entre os bicos e concentrações do líquido, obteve-se um padrão de distibuição. $\mathrm{O}$ melhor padrão $\mathrm{e}$ justamente aquele que mantem uma uniformidade maior, isto e, o que apresenta menor amplitude entre os pontos de maior e menor deposição. Assim, para calda com $15 \%$ de glyphosate e vazão de $0,431 / \mathrm{min}$, a melhor distância entre bicos foi de 1,40 $\mathrm{m}$ (figura $2 \mathrm{E}$ ) ao passo que para a calda com $7,5 \%$ e glyphosate e vazão de $0,961 / \mathrm{min}$, a melhor distância entre os bicos foi de 1,80. $\mathrm{m}$ (Figura 1B). As figuras mostram que a concentração influi grandemente no padrão de distribuição, e portanto, e imprescin- dível que para cada concentração a ser usada, se faça um estudo em mesa de deposição para se conhecer o padrão de distribuição. Em condições de campo, a não observação desses resultados pode levar à uma ação ineficiente ou desperdício do produto, pois, corre-se o risco do produto ser colocado em altas doses em determinados locais e em baixas doses em outros, o que implica diretamente numa desuniformidade de aplicação com conseqüências na eficiência da ação do produto $(10,3)$.

Portanto, a determinação da distância entre bicos que proporcione o melhor padrão de distribuição e fundamental para a correta utilização dessa técnica.

Essa determinação e passo imprescindível e tanto fabricante do bico como as indústrias de produtos químicos devem se preocupar em efetuar tais estudos de forma a fornecer ao usuário o parâmetro correto para a utilização desses bicos. Considerando-se que se trata de uma nova técnica em fase de introdução, a inobservância desses cuidados pode comprometer seria e definitivamente a sua aceitação.

Para assegurar que no campo seja reproduzido o padrão fixado em laboratório e interessante a utilização da barra auto-estável (13), para que as oscilações da mesma sejam mantidas dentro de limites aceitáveis, não interferindo assim na distribuição previamente estabelecida. 

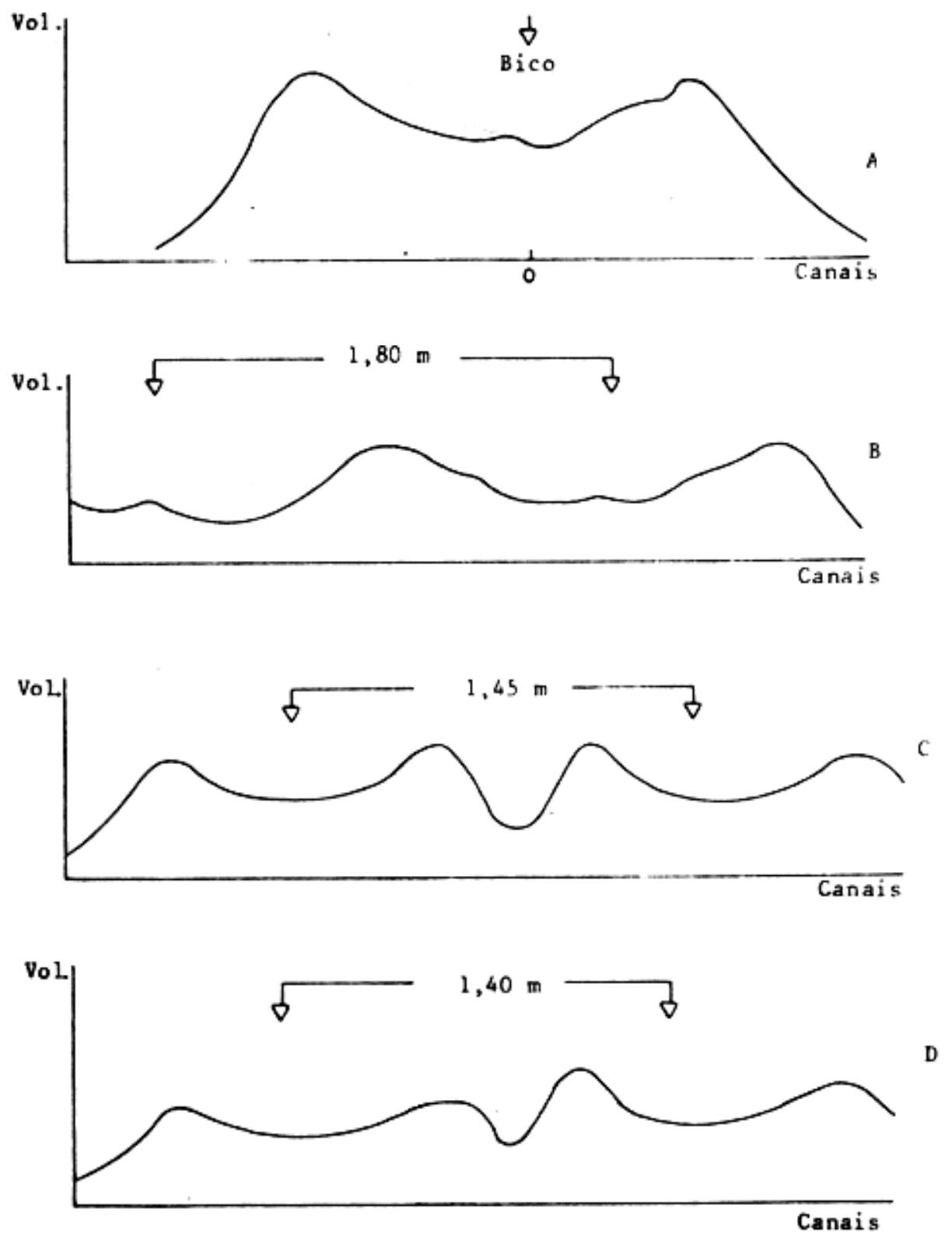

FIGURA 1 - Padrão de deposição de bicos Micromax operado a 1600 rpm, apli cando formulação comercial de glyph osate a 7,5\% em água .

$\mathrm{A}=\mathrm{Um}$ bico, com vazão de $0,96 \mathrm{Z} / \mathrm{min}$;

$\mathrm{B}=$ Dois bicos, com vazão de $0,967 / \mathrm{min}$ cada, espaçados $1,80 \mathrm{~m}$ entre si;

$\mathrm{C}=$ Dois bicos, com vazão de 0,43 Z/min cada, espaçados 1,45 m entre si;

$\mathrm{D}=$ Dois bicos, com vazão de 0,43 1/min cada, espaçados 1,40 m entre si. 

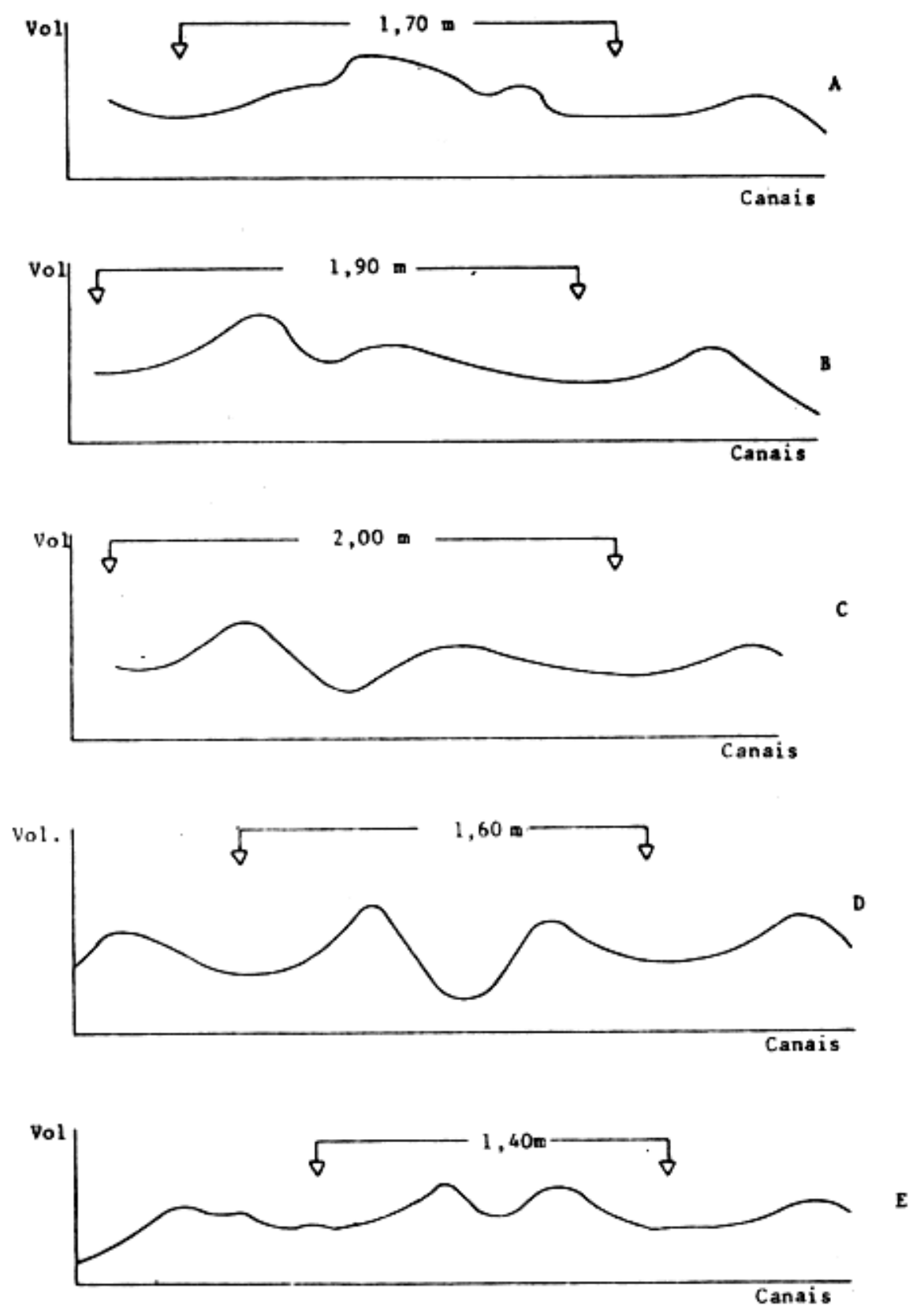

FIGURA 2 - Padrão de deposição de bicos Micromax operando a 1600 rpm, aplicando formulação comercial de glyphosate diluído a $15 \%$ de agua.

A = Dois bicos, com vazão de 0,96 7/min cada, espaçados $1,70 \mathrm{~m}$ entre si; $\mathrm{B}=$ Dois bicos, com vazão de $0,961 / \mathrm{min}$ cada, espaçados $1,90 \mathrm{~m}$ entre si; $\mathrm{C}=$ Dois bicos, com vazão de 0,96 1/min cada, espaçados $2,00 \mathrm{~m}$ entre si; $\mathrm{D}=$ Dois bicos, com vazão de $0,43 \mathrm{Z} / \mathrm{min}$ cada, espaçados $1,60 \mathrm{~m}$ entre si; $\mathrm{E}=$ Dois bicos, com vazão de 0,43 //min cada, espaçados $1,40 \mathrm{~m}$ entre si. 

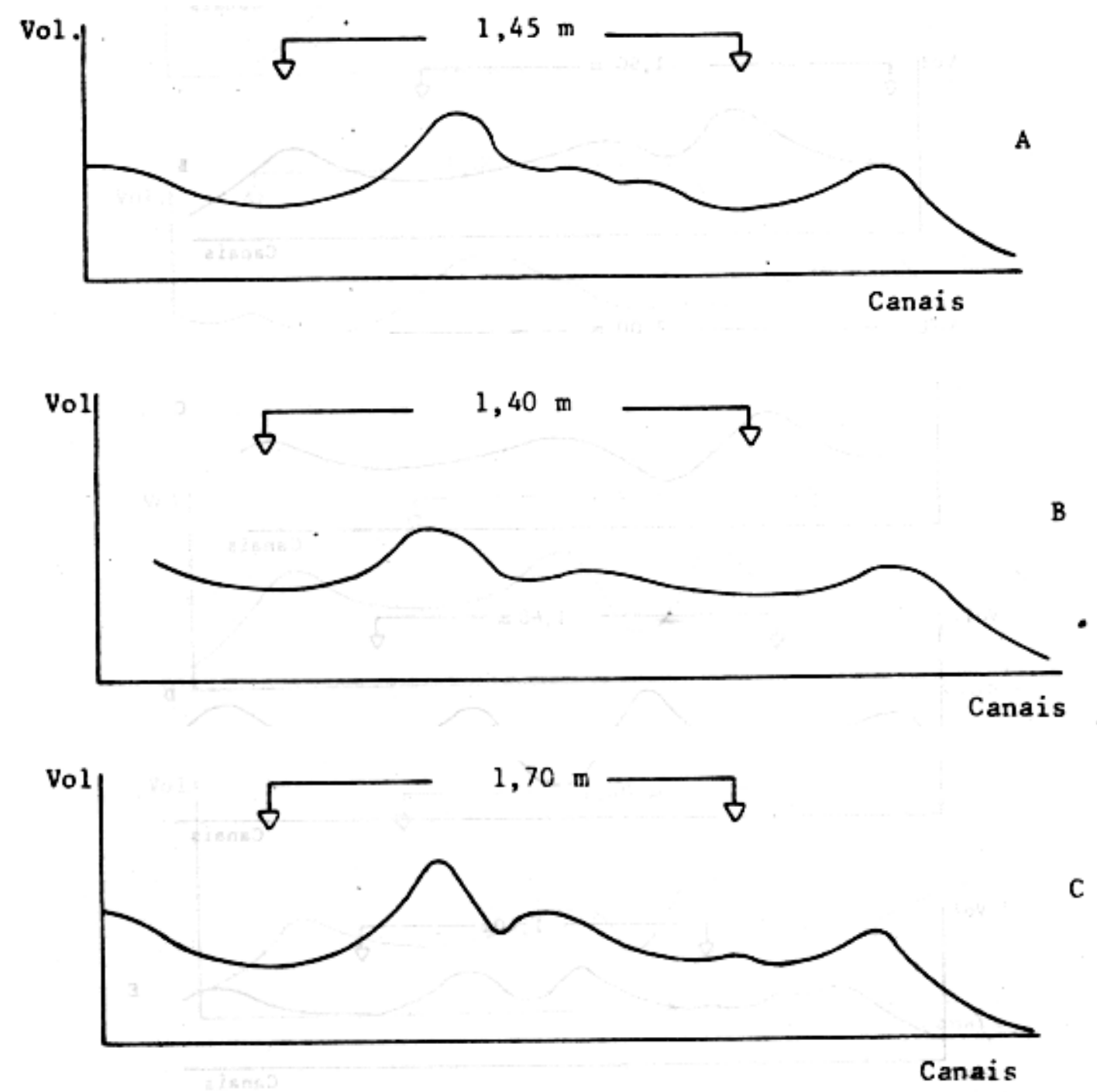

FIGURA 3 - Padrão de deposição de bicos Micromax operando a 1600 rpm com vazão de $0,43 / / \mathrm{min}$, aplicando formulação comercial de gly pho sate diluído em agua, em duas concentrações.

$\mathrm{A}=$ Dois bicos espaçados de $1,45 \mathrm{~m}$ e concentração de $4 \%$;

$\mathrm{B}=$ Dois bicos espaçados de $1,40 \mathrm{~m}$ e concentração de $4 \%$;

$\mathrm{C}=$ Dois bicos espaçados de 1,70 e concontração de $2 \%$. 
QUADRO 1 - Tamanho e uniformidade das gotas produzidas pelo bico Micro max a $1600 \mathrm{rpm}$, aplicando diferentes concentrações de glypho sate, em dois regimes de vazão.

\begin{tabular}{|c|c|c|c|c|}
\hline \multirow{2}{*}{ Concentração* } & \multirow{2}{*}{$\begin{array}{l}\text { Vazão } \\
1 / \text { min }\end{array}$} & \multicolumn{2}{|c|}{ Tamanho de gotas $(\mu \mathrm{m})$} & \multirow{2}{*}{$\mathrm{r}^{* *}$} \\
\hline & & vmd & nmd & \\
\hline $4 \%$ & 0,96 & 292 & 243 & 1,21 \\
\hline $5 \%$ & 0,96 & 267 & 183 & 1,47 \\
\hline $6 \%$ & 0,96 & 282 & 220 & 1,31 \\
\hline $9 \%$ & 0,43 & 238 & 173 & 1,40 \\
\hline $11 \%$ & 0,43 & 225 & 181 & 1,24 \\
\hline $13 \%$ & 0,43 & 233 & 188 & 1,24 \\
\hline
\end{tabular}

* - Concentração da formulação comercial;

** - Coeficiente de dispersão $(\mathrm{r}=\mathrm{v} \mathrm{md} / \mathrm{nmd})$.

Em relação ao tamanho e uniformida de das gotas (Quadro 1) pode-se notar que para a vazão mais baixa elas são menores. Estes dados estão de acordo com outros autor es $(9,7,6,2,12)$. Essa variação do taman ho de gotas explica porque na vazão de $0,96 \mathrm{Z} / \mathrm{min}$. a distancia ótima deve ser maior que para a vazão de $0,43 / / \mathrm{min}$. Gotas maiores, dotadas de maior massa, são arremessadas pelo disco com maior energia cinética e são capazes de vencer distância maior no ar e vice-versa.

Em media, o vmd das gotas produ zidas no regime de vazão de 0,96 //min foi de 280 um. Considerando se que o bico foi projetado para produzir gotas de $250 \mu \mathrm{m}$ a $2000 \mathrm{rpm}$ e vazão de 1 1/min, os valores observados estão dentro do esperado. O coeficiente de dispersão (r) situou-se dentro dos limites para a especificação do método CDA.

\section{LITERATURA CITADA}

01. Adas (Agricultural Division Advisory Service) Controlled droplet application of agricultural chemical. Northumberland Ministry of Agriculture Fisheries and Food. Inglaterra, 1981. $11 \mathrm{p}$.

02. Bals,E.J. The reasons for C.D.A. (Controlled drop application). In: BRITSH CROP PROTECTION CONFERENCE - WEEDS, Brighton, 1978. Proceedings. p.101-106.

03. Bode, L.E. \& Butle,B.J. Spray chacacteristics of rotary atomizer. Pesticide Formu- 
tion and application

Systems: Seconc Conference, ASTM STP 795, Kg Seymour,

Ed. American Society for Tes ting and materials, Philadel phia, 1983. p. 89-104.

04. Combellack,J.H. The value of controlled droplet application (C.D.A.) as spot spray technique for the ccntrol of noxious weeds in Victória.

In: CONFERENCE OF THE CCUNCIL OF AUSTRALIAN WEED SCIEN CE SOCIETIES, 5 Victória, 1978. Proceedings 15-24.

05. Combellack,J.H. \& Harris, R.V. Field trials with CDA spot sprayers. In: CONFERENCE OF THE COUNCIL OF AUSTRALIAN WEED SCIENCE SOCIETIES, 5\%, Victória, 1978. Proceedings. p. 39-49.

06. Heijne,C.G. A study of the effects of disc speed and flow rate on the performance OF THE Micron Battleship.In: BRITISH CROP PROTECTION CCNFERENCE WEEDS, Brighton, 1978. Proceedings. p.673-679.

07- Johnstone,D.R. the influence of physical anc metecrological factors on the deposition and drif of spray droplets of controlled size: In:
SYMPOSIUM ON CCNTROLLED DROP APPLICATION, Reading 12th13th April, 1978. Proceedings. p.43-57

o\&. Johnstone,D.R. Statistical description of spray drop size for controlled drop applica tion. In: SYMPOSIUM ON CONTROLLED DROP APFLICATION, Reading, 12th-13th, April, 1978. Proceedings, p.7-21.

09. Lake,J.R.; Frost,A.R.; Green,R. Measurementes of drop size and spray distribution from a Micron Herbi disc. In: BRI TISH CROP PROTECTION CONFERENCE WEEDS, Brighton. 1976. Proceedings. v.2, p. 399-405.

10. Lavers,A. \& Stovell,F.R. A review of broad-leaved weed and wild-oat herbicides at reduced volume rates. - In: BRITISH CROP PROTECTION CCNFERENCE-WEEDS Brighton, 1978. Prcceedings. p. 717-728.

11. Lush, G.B. Preface.In: SYMPOSIUM ON CCNTROLLED DROP APFLICATION, Readin, 12th 13th April, 1978. Proceedings. 12. Matthews,G.A. Pesticide applica tion me thods. Londres, Longman, 1979. 325p.

13. Nation,H.J. The preference and stability of spray bocms.In: SPFAYING SYSTEMS FCR THE $1980^{\prime}$ s. p. $145-158,1980$. 